DOI: https://doi.org/10.46296/gt.v3i6.0014

\title{
PROGRAMA AUDIOVISUAL DE TÉCNICAS DE HIGIENE BUCAL PARA LA FACULTAD DE ODONTOLOGÍA DE LA ULEAM, ECUADOR
}

\section{AUDIOVISUAL PROGRAM OF ORAL HYGIENE TECHNIQUES FOR THE FACULTY OF DENTISTRY OF ULEAM, ECUADOR}

\author{
Zambrano-Pincay María Victoria $^{1 *}$ \\ ${ }^{1}$ Universidad Laica Eloy Alfaro de Manabí, ULEAM. Manta, Ecuador.
}

*Correo: mzambrano-pincay19@gmail.com

\begin{abstract}
Resumen
La presente investigación se realiza en la Facultad de Odontología de la Universidad Laica Eloy Alfaro de Manabí, con el objeto de apoyar en la planificación y actividades educativas que tienen como fin capacitar a pacientes y a sus acompañantes que esperan ser atendidos en el Centro de atención en la Facultad de Odontología de la ULEAM, para adquirir comportamientos que favorezcan la salud bucodental a través de un programa audiovisual de técnicas de higiene bucal. Este estudio es de tipo descriptivo, transversal y analítico. Se hace referencia a los programas preventivos promocionales de salud bucal, con revisión de conceptos de los determinantes de la salud, prevención y promoción. Donde se concluye que la técnica audiovisual se inserta en una metodología que desarrolla a los participantes como sujetos activos, capaces de construir nuevos conocimientos; el programa que se desarrolla se espera contribuya al mejoramiento de la salud oral.
\end{abstract}

Palabras clave: Programa, audiovisual, técnicas, higiene, bucal.

\begin{abstract}
This research is carried out at the Faculty of Dentistry of the Laica Eloy Alfaro University in Manabí, in order to support educational planning and activities that aim to train patients and their companions who expect to be cared for at the Health Care Center in the ULEAM School of Dentistry, to acquire behaviors that promote oral health through an audiovisual program of oral hygiene techniques. This study is descriptive, cross-sectional and analytical. Reference is made to promotional preventive oral health programs, with a review of concepts of the determinants of health, prevention and promotion. Where it is concluded that the audiovisual technique is inserted into a methodology that develops the participants as active subjects, capable of building new knowledge; The program being developed is expected to contribute to the improvement of oral health.
\end{abstract}

Keywords: Program, audiovisual, techniques, hygiene, oral.

Información del manuscrito:

Fecha de recepción: 20 de marzo de 2020

Fecha de aceptación: 09 de junio de 2020

Fecha de publicación: 10 de julio de 2020 


\section{Introducción}

Según los registros de la Organización Mundial de la Salud (OMS, 2019) se estima que la caries de los dientes permanentes es la más común de todas las afecciones evaluadas, con 2,4 billones de personas en todo el mundo que sufren caries de dientes permanentes y 486 millones de niños con caries de dientes primarios.

Por consiguiente, se requiere acción y participación del sector público asociado directamente con la responsabilidad de establecer políticas de salud pública para establecer un programa que permita aportar una ingesta suficiente de flúor en las áreas donde esto carece, para minimizar la prevalencia de caries dental. Evidentemente, esta misiva puede ser abordada a través de la fluoración del agua potable o, cuando esto no sea posible, mediante la fluoración del cloruro de sodio o la leche, así mismo, se proponen estrategias relacionadas con el uso de productos de cuidado dental que contengan fluoruro, y al recomendar una dieta baja en azúcar (Espinoza-Usaqui, E. M., \& PachasBarrionuevo, 2013; Kim et al., 2017).
A nivel mundial, la reducción de la prevalencia de caries dental en la se ha debido a la amplia exposición a diversas fuentes de fluoruro (Mutis et al., 2019; Tinanoff \& Douglass, 2001).

Una de las principales funciones $y / 0$ competencias de las Ciencias Odontológicas está relacionada con la prevención de las enfermedades de la cavidad bucal. No obstante, se exponen que han sido pocos los estudios que han evaluado los patrones de hábitos de higiene bucal en niños a principios de edad. Por lo cual se asocia la edad y el estatus socio-económico con la práctica de hábitos de higiene bucal (Ismail, 1998).

Por otra parte, es imprescindible incorporar las tecnologías de la información como técnica audiovisual que proponga consejos referentes a las técnicas de higiene oral.

La presente investigación es importante y beneficiosa para la comunidad universitaria de la Facultad de Odontología de la Universidad Laica Eloy Alfaro de Manabí (ULEAM), puesto que se sustenta en un programa audiovisual, debido a que la 
sociedad está vinculada con la parte tecnológica, mediante el cual la información se enfoca de manera clara y precisa; el programa es de mucha importancia y trata de solucionar uno de los grandes problemas odontológicos que afecta a la sociedad, por lo que su impulso y aplicación toma mayor envergadura.

\section{Metodología}

\subsection{Tipo de estudio}

El presente estudio se realizó mediante las metodologías de tipo descriptivo, transversal y analítico.

Descriptivo. Se pretendió especificar las propiedades importantes de personas, grupos, comunidades 0 cualquier otro fenómeno que sea sometido a análisis. Permitió medir o evaluar diversos aspectos, dimensiones o componentes del fenómeno a investigar y con ello demostrar la funcionalidad de un programa audiovisual sobre técnicas de higiene bucal; además, permitió ayudar a reconocer las circunstancias y características del objeto de estudio, identificando así el problema.

Transversal. Se recolectaron datos en un solo momento, en un tiempo único. Su propósito fue describir variables y analizar su prevalencia en un momento dado.

Analítico. Tuvo como propósito medir el grado de relación que existe entre dos o más conceptos o variables.

\subsection{Diseño de investigación}

La investigación se enmarcó en una investigación de carácter:

- Descriptivo, puesto que ayudó a identificar las características del problema detectado, permitiendo detallar diferentes aspectos de las técnicas de higiene bucal y en lo referente a un programa audiovisual que se exhibirá en el Centro de Atención Odontológica de la ULEAM, mientras los pacientes y sus acompañantes esperan ser atendidos en el Centro de Atención Odontológica, con el fin de lograr el aprendizaje por parte de los pacientes tanto como de sus padres para poderlo aplicar en casa.

- Bibliográfica, permitió orientar en la revisión de textos y fuentes bibliográficas, con lo cual se logró estructurar y desarrollar el marco teórico, referencial y conceptual. 
- De campo, corresponde al trabajo que se realizó en la institución o lugar donde se detectó el problema, consecuentemente, se requiere de alguna manera darle una solución, la cual se describe en la propuesta del proyecto investigativo.

\subsection{Técnicas de redacción de} datos

Para el procedimiento de los resultados y el análisis estadístico se establece lo siguiente:

- El tipo de información obtenida fue de tipo cualitativo, debido a que la investigación está referida a todos aquellos aspectos que denotan cualidad y que son susceptibles de tomar valores no numéricos, diferentes, comprendidos o no dentro de cierto límite, siendo el objetivo la riqueza, profundidad y calidad de la información y no la cantidad y estandarización.

- La presente investigación utilizó como métodos generales el análisis y la deducción, pues se verifica la información obtenida de fuentes primarias $y$ secundarias; se pretende deducir la aplicación de las teorías en el aspecto de la comunicación formativa, mediante la implementación de un programa audiovisual. Para tal fin, el método específico empleado es el documental o revisión bibliográfica de la teoría.

\section{Resultados y discusión}

En concordancia con el Plan Nacional de Salud Bucal, los profesionales del área de Odontología deben tener como enfoque primordial la promoción (creación de políticas y protocolos adecuados para reducir los índices de incidencia de las enfermedades estomatológicas) y la prevención (primaria, secundaria y terciaria) de la salud oral en los individuos de las comunidades.

La creación de programas de promoción de la salud oral debe direccionarse hacia el desarrollo de: i) comportamientos saludables ( limpieza adecuada de dientes y encías, correcta aplicación de la técnica de cepillado, control de placa bacteriana por parte de los individuos y los profesionales en una revisión periódica del estado de salud bucal, uso de hilo dental como accionar complementario y 
obligatorio) y ii) capacitación para el conocimiento de la importancia de la lactancia materna y la seguridad alimentaria en los aspectos de nutrición (generando mejoras en el sistema inmunitario y un refuerzo en el aspecto biológico) y de dietas bajas en agentes cariogénicos, lo cual consecuentemente reduce la prevalencia de estos casos.

El trabajo de los profesionales de la salud oral también debe sustentarse en la obligación de dirigirse hacia la prevención primaria, en aras de contrastar las patologías antes de su detección en revisiones periódicas en los consultorios odontológicos de los servicios públicos sanitarios, en lo cual se debe hacer una detección temprana de la placa bacteriana, enseñar el correcto procedimiento del cepillado dental y de ser necesario la aplicación de sellantes, todo esto sustentando en un diagnóstico temprano y por lo tanto sin permitir la cronicidad de las enfermedades estomatológicas, por lo cual es imperioso aplicar la profilaxis bucal por parte de los profesionales pertinentes (CabezaBernhardt et al., 2016).

Se sugiere que el profesional odontológico realice un seguimiento permanente del estado bucal de los pacientes, de modo que pueda realizar informes dirigidos a los centros hospitalarios o sanitarios donde se realiza la vigilancia epidemiológica de estos pacientes.

Además, Socorro et al., (2007) no determinaron diferencias estadísticamente significativas cuando se compararon los datos relacionados con la presencia del hábito de salud bucal y el nivel socioeconómico ( $p>\quad 0,05)$ La relación reportada entre las variables mencionadas se puede fundamentar a partir de la edad de los niños durante una etapa de vida en la que están aprendiendo acerca de los hábitos para el cuidado de la cavidad bucal.

Por otra parte, el tipo de educación también tiene incidencia en el nivel de salud bucodental, de modo que en las escuelas públicas se presenta un nivel de salud dental más bajo y un número bajo de madres de familia con altos conocimientos en higiene bucal; en contraste con las escuelas privadas, donde los niños reflejan un comportamiento opuesto a sus pares de instituciones de carácter público (Ferreira-Gaona et al., 2016). 
Finalmente, se genera una propuesta basada en un programa audiovisual de técnicas de salud oral, expuesta en la tabla 1.

Tabla 1. Programa audiovisual de técnicas de higiene bucal

\begin{tabular}{|c|c|c|c|}
\hline COMPONENTES & \multicolumn{3}{|c|}{ DESCRIPCIÓN } \\
\hline Propósitos & \multicolumn{3}{|c|}{$\begin{array}{l}\text { Conservar en buen estado de salud la boca y los dientes y evitar las enfermedades } \\
\text { bucodentales, sobre todo la caries y los problemas de encías que son muy frecuentes y, sin } \\
\text { embargo, fácilmente prevenibles si se aplican diariamente los cuidados adecuados en cada } \\
\text { edad. }\end{array}$} \\
\hline Objetivos & \multicolumn{3}{|c|}{$\begin{array}{l}\text { Apoyar en el quehacer educativo de capacitar a pacientes y sus acompañantes que esperan } \\
\text { ser atendidos en el Centro de Atención en la facultad de odontología de la Universidad Laica } \\
\text { Eloy Alfaro de Manabí, para adquirir comportamientos que favorezcan la salud bucodental a } \\
\text { través de un programa audiovisual de técnicas de higiene bucal }\end{array}$} \\
\hline Metas & \multicolumn{3}{|c|}{$\begin{array}{l}\text { El programa de higiene bucal tiene como meta mejorar la salud bucodental a la población } \\
\text { que acude al Centro de Atención en la facultad de odontología de la Universidad Laica Eloy } \\
\text { Alfaro de Manabí, previniendo la aparición de los problemas más frecuentes: caries, } \\
\text { enfermedad periodontal, etc. }\end{array}$} \\
\hline $\begin{array}{l}\text { Técnicas y } \\
\text { Estrategias }\end{array}$ & \multicolumn{3}{|c|}{$\begin{array}{l}\text { 1. Informar a los asistentes sobre técnicas de higiene bucal (alimentación no cariógena, } \\
\text { cepillado correcto, etc.) a través del programa audiovisual } \\
\text { 2. Orientar a los asistentes sobre las técnicas de higiene bucal que se pueden realizar para } \\
\text { prevenir las patologías orales más frecuentes. } \\
\text { 3. Capacitar a los docentes, alumnos y la población que acude al Centro de Atención en la } \\
\text { facultad de odontología de la Universidad Laica Eloy Alfaro de Manabí sobre técnicas de } \\
\text { higiene bucal. }\end{array}$} \\
\hline Actividades & $\begin{array}{l}1.1 . \\
1.2 . \\
1.3 . \\
1.4 . \\
1.5 . \\
1.6 . \\
1.7 . a) \\
1.7 . \text { b) }\end{array}$ & \multicolumn{2}{|c|}{$\begin{array}{l}\text { Reunión Maestrante (director) } \\
\text { Solicitar autorización } \\
\text { Diseñar el programa } \\
\text { Autorización para ejecutar el programa } \\
\text { Socializar el programa } \\
\text { Aplicación del programa a los asistentes } \\
\text { Evaluación de resultados, que se da al final del programa. } \\
\text { Evaluación del proceso, que se realiza durante la ejecución del programa }\end{array}$} \\
\hline Recursos & \multicolumn{3}{|c|}{$\begin{array}{l}\text { - Humanos } \\
\text { - Materiales: Oficio, Cámara fotográfica, Papel, Computadora, Impresora, Insumos de } \\
\text { oficina, Internet, TV, Memoria Flash; DVD, CD }\end{array}$} \\
\hline Ámbitos & \multicolumn{3}{|c|}{$\begin{array}{l}\text { El programa se implementará en el Centro de Atención en la Facultad de Od0ontología de } \\
\text { la Universidad Laica Eloy Alfaro de Manabí }\end{array}$} \\
\hline $\begin{array}{l}\text { Beneficiarios } y / 0 \\
\text { Destinatarios }\end{array}$ & \multicolumn{3}{|c|}{$\begin{array}{l}\text { Participación de Docentes, alumnos y la población en general que acude al Centro de } \\
\text { Atención en la Facultad de Odontología de la Universidad Laica Eloy Alfaro de Manabí }\end{array}$} \\
\hline Fases o etapas & \multicolumn{3}{|c|}{$\begin{array}{l}\text { - Registro de asistencia } \\
\text { - Evidencias fotográficas } \\
\text { - Evaluación del programa }\end{array}$} \\
\hline \multirow{8}{*}{ Cronograma } & \multicolumn{3}{|c|}{ Programa audiovisual de técnicas de higiene bucal } \\
\hline & 1.1. & Reunión Maestrante (director) & Septiembre 20- 2017 \\
\hline & 1.2. & Solicitar autorización & Octubre 1-2017 \\
\hline & 1.3. & Diseñar el programa & Septiembre 20- 2017 \\
\hline & 1.4. & $\begin{array}{l}\text { Autorización para ejecutar el } \\
\text { programa }\end{array}$ & Octubre 8-2017 \\
\hline & 1.5. & Socializar el programa & Octubre 8-2017 \\
\hline & 1.6. & $\begin{array}{l}\text { Aplicación del programa a los } \\
\text { asistentes }\end{array}$ & Octubre 11-2017-Diciembre11-2017 \\
\hline & 1.7. & Evaluación del programa & Diciembre 11-2017 \\
\hline Responsables & \multicolumn{3}{|c|}{ Maestrante(director) y alumno } \\
\hline
\end{tabular}

\section{Conclusiones}

\section{El programa desarrollado se} encuentra dirigido a pacientes y sus acompañantes, durante el lapso de tiempo de espera hasta ser atendidos en el Centro de Atención en la Facultad de Odontología de la Universidad Laica Eloy Alfaro de 
Manabí. Este programa se refiere particularmente a las técnicas de higiene bucal y su contribución a la mejora de la salud oral.

La implementación de un programa audiovisual de técnicas de higiene bucal dirigido a pacientes y sus acompañantes que esperan ser atendidos en el Centro de Atención en la Facultad de Odontología de la Universidad Laica Eloy Alfaro de Manabí pretende reducir la falta de cultura en los cuidados de salud oral, como principal causa para el deterioro de la salud en los ecuatorianos.

\section{Bibliografía}

Cabeza-Bernhardt, G., GonzálezAndrade, F., \& ParedesAndrade, C. (2016). Estado de salud oral en el Ecuador. Revista OACTIVA UC Cuenca, 1(3), 65-70.

Espinoza-Usaqui, E. M., \& PachasBarrionuevo, F. (2013). Programas preventivos promocionales de salud bucal en el Perú. Revista Estomatológica Herediana, 23(2), 101-101.

Ferreira-Gaona, M. I., DíazReissner, C. V., Pérez, N.,
Sanabria-Vázquez, D. A., Alvarenga-Rodas, M. V., Bazán-Cohene, D. D., ... \& López, M. (2016). Salud bucal en preescolares y nivel de conocimiento sobre higiene bucal de sus madres, en escuelas públicas y privadas de San Lorenzo, Paraguay. Pediatría (Asunción): Órgano Oficial de la Sociedad Paraguaya de Pediatría, 43(2), 129-136.

Ismail, A I. (1998). Prevención de la caries en la primera infancia, Comunidad. Odontología y Epidemiología Oral. Vol. 26, Suplemento 1, pág. 49-61.

Kim, H. N., Kim, J. H., Kim, S. Y., \& Kim, J. B. (2017). Associations of community water fluoridation with caries prevalence and oral health inequality in children. International journal of environmental research and public health, 14(6), 631. doi: 10.3390/ijerph14060631

Mutis, M. J., Chamut, S., Morón, E., \& Peixoto, C. D. (2019). Status of the Epidemiological Surveillance Systems for Salt and Water Fluoridation 
Programs in Latin America

and the Caribbean.

Universitas Odontologica,

38(80).

doi:

10.11144/Javeriana.uo38-

80.sess

OMS. (2019). Fluoruro inadecuado o en exceso. Ginebra: Organización Mundial de la Salud. Disponible en: https://www.who.int/ipcs/asse ssment/public_health/fluoride/ en/
Socorro, M., Gómez, D., Torres, J., Acevedo, A. M., \& RojasSánchez, F. (2007). Hábitos de higiene bucal y el nivel socio-económico de niños entre 15 y 20 meses de edad. Acta Odontológica Venezolana, 45(2).

Tinanoff N., \& Douglass, J. M. (2001). ¿Toma de decisiones clínicas para el manejo de caries en dientes primarios?. Revista de Educación Dental, 65(10), 1133-1142. 